\title{
4D Minimal Non-simple Sets
}

\author{
C.J. Gau ${ }^{1}$ and T. Yung Kong ${ }^{2}$ \\ 1 Department of Computer Science \\ Graduate School and University Center \\ City University of New York \\ New York, NY 10036, U.S.A. \\ cgau@lagcc. cuny.edu \\ ${ }^{2}$ Department of Computer Science \\ Queens College \\ City University of New York \\ Flushing, NY 11367, U.S.A. \\ ykong@cs.qc.edu
}

\begin{abstract}
One method of verifying that a given parallel thinning algorithm "preserves topology" is to show that no iteration ever deletes a minimal non-simple ("MNS") set of 1's. The practicality of this method depends on the fact that few types of set can be MNS without being a component. The problem of finding all such types of set has been solved (by Ronse, Hall, Ma, and the authors) for 2D and 3D Cartesian grids, and for 2D hexagonal and 3D face-centered cubic grids. Here we solve this problem for a $4 \mathrm{D}$ Cartesian grid, in the case where 80 -adjacency is used on 1's and 8-adjacency on 0's.
\end{abstract}

Keywords. 4D xel, attachment, minimal non-simple, MNS, parallel thinning.

\section{Introduction}

Loosely speaking, a set of 1's in a binary image is said to be simple if parallel deletion of those 1's "preserves topology". A non-simple set of 1's must contain a minimal non-simple (MNS) set. So one way to prove that a given parallel thinning algorithm always "preserves topology" is to show that no iteration of that algorithm can ever delete an MNS set of 1's.

The practicality of this method depends on the fact that there are few types of possible MNS sets, and even fewer types of sets that can be MNS without being components of the 1's. (Here we consider two sets of grid points to be of the same type if one set is a translate of the other.)

The problem of identifying all such types of sets has been solved for $2 \mathrm{D}$ and 3D Cartesian grids, and for 2D hexagonal and 3D face-centered cubic grids. Ronse [1] introduced the concept of an MNS set (called an MND set in 1]), and solved the problem for a 2D Cartesian grid with $(8,4)$ or $(4,8)$ connectedness. Hall 2] solved the problem for a 2D hexagonal grid with $(6,6)$ connectedness. Ma [3] solved the problem for a 3D Cartesian grid with $(26,6),(6,26),(18,6)$, or 
$(6,18)$ connectedness. The authors 4 solved the problem for a $3 \mathrm{D}$ face-centered cubic grid with $(18,12),(12,12)$, or $(12,18)$ connectedness.

In this paper we solve the problem for a $4 \mathrm{D}$ Cartesian grid with $(80,8)$ connectedness: We determine which sets of grid points can be MNS, and also determine which of those sets can be MNS without being a component of the 1's. This gives a method of verifying that a given 4D parallel thinning algorithm always "preserves topology". Such algorithms might be of use in temporal image analysis, in which a time sequence of 3D images is represented as a 4D image.

As in [4] and [5], we do not work directly with grid points, but prefer to think in terms of their Voronoi neighborhoods. For a 4D Cartesian grid the Voronoi neighborhoods of grid points are "upright" 4-dimensional hypercubes. We will call such a hypercube a 4 -xel. Assuming without loss of generality that the grid points have coordinates of the form $\left(i_{1}+0.5, i_{2}+0.5, i_{3}+0.5, i_{4}+0.5\right)$ where the $i$ 's are integers, the vertices of a 4 -xel have integer coordinates and the edges of a 4 -xel have length 1.

We identify each binary image on a 4D Cartesian grid with the set of all 4 -xels that are centered at the grid points which have value 1 in the image. This allows us to define a binary image simply as a finite set of 4 -xels. The concepts of a simple 1 and a simple set of 1's will be made precise by the concepts of a simple 4-xel and a simple set of 4-xels (in a binary image).

We will use the same general approach to MNS sets as was used in 4] and [5]. Our work will be based on a characterization of simple 4-xels in terms of their attachment sets that was given in [6]. The attachment set of a 4-xel in a binary image is the subset of the boundary of the 4-xel that is shared with other 4-xels in the image.

\section{$2 n$-Xels, 4D Binary Images, Xel-Complexes}

An elementary 0 -cell is a singleton set $\{i\}$, where $i$ is an integer. An elementary 1 -cell is a closed unit interval $[i, i+1]$ of the real line, where $i$ is an integer. A $4^{-}$ $x e l$ is a Cartesian product of 4 elementary 1-cells. More generally, for $0 \leq n \leq 4$ an $n$-xel is a Cartesian product of $n$ elementary 1-cells and $4-n$ elementary 0 -cells, in some order. If $q$ is an $n$-xel for some $n$, then we say $q$ is a $x e l$. A 0 -xel will also be called a vertex and a 1-xel will also be called an edge. A $4 D$ binary image is a finite set of 4 -xels.

An $n$-xel is the trajectory of an $(n-1)$-xel as it moves one unit in the positive or negative direction of the coordinate axis that is perpendicular to the $(n-1)$ xel. This is illustrated in Figure 1 The reader may sometimes find it helpful to think of 4-xels as trajectories of 3 -xels that move through a unit of time.

If a xel $y$ is a proper subset of a xel $x$, then we say $y$ is a proper face of $x$, and write $y<x$. A xel $x$ is a Cartesian product of elementary 1-cells and elementary 0 -cells, and each proper face of $x$ can be obtained from the product by replacing one or more of its elementary 1-cells $[i, i+1]$ with $\{i\}$ or $\{i+1\}$. A set $K$ of xels is called a xel-complex if $K$ is finite and, for every xel $x \in K$, every proper face of $x$ is also in $K$. If $y<x$ is a $k$-xel then we say $y$ is a $k$-face of $x$. 


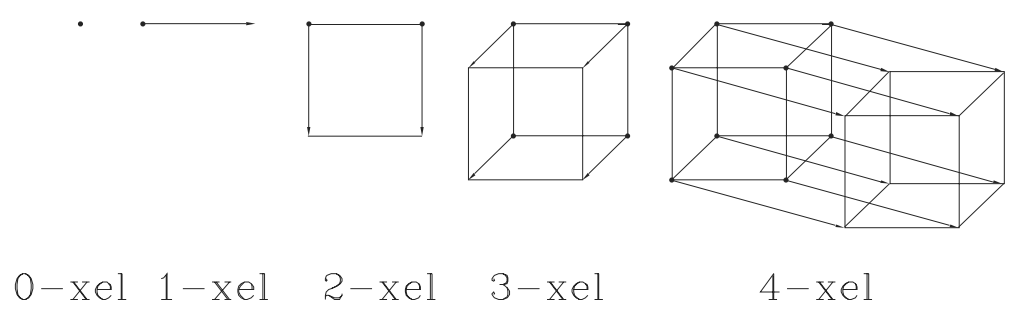

Fig. 1. An $n$-xel can be viewed as the trajectory of a moving $(n-1)$-xel.

Each 4-xel intersects just 80 other 4-xels, and shares a 3-face with just 8 other 4-xels. Accordingly, two 4-xels are said to be 80-adjacent if they are distinct but intersect, and are said to be 8 -adjacent if their intersection is a 3 -xel.

For any set $S$ of 4 -xels, and $\kappa=80$ or 8 , two 4 -xels $q_{1}, q_{2} \in S$ are said to be $\kappa$-connected in $S$ if they are related by the reflexive transitive closure of the $\kappa$ adjacency relation on $S$. This is an equivalence relation on $S$, and its equivalence classes are called the $\kappa$-components of $S$.

\section{Simple 4-Xels and Attachment Sets of 4-Xels}

A 4-xel in a $4 \mathrm{D}$ binary image $I$ is said to be simple in $I$ if (loosely speaking) its deletion "preserves topology". More precisely, if $I$ is a 4D binary image we say that a 4-xel $q \in I$ is simple if the polyhedron $\bigcup(I \backslash\{q\})$ is a strong deformation retract of the polyhedron $\bigcup I$. In other words, $q$ is simple if and only if the union of the 4-xels in $I$ can be continuously deformed over itself onto the union of the 4-xels in $I \backslash\{q\}$, in such a way that all points in $\bigcup(I \backslash\{q\})$ at the start remain fixed throughout the deformation process.

This definition of simpleness in terms of the polyhedra $\bigcup I$ and $\bigcup(I \backslash\{q\})$ is consistent with the use of 80-connectedness as the definition of connectedness on the 4-xels in $I$ and the use of 8-connectedness as the definition of connectedness on the 4 -xels in the complement of $I$. Indeed, two 4-xels belong to the same 80 -component of $I$ if and only if they lie in the same connected component of $\bigcup I$, and they belong to the same 8-component of the 4-xels in the complement of $I$ if and only if their interiors lie in the same connected component of $\mathbb{R}^{4} \backslash \bigcup I$.

Our definition of simpleness has the advantage of being independent of dimensionality and of the shapes of xels. (For example, the same definition of simpleness could be used in 3D binary images on a face-centered cubic grid, except that instead of 4 -xels we would have dodecahedral rhomboids belonging to a tessellation of 3-space by congruent dodecahedral rhomboids. In fact our definition of simpleness would be appropriate even in 5- and higher-dimensional binary images.) The definition involves continuous deformation, but Theorems 1 and 2 below give essentially discrete sets of necessary and sufficient conditions for $q$ to be simple in $I$. 
These two theorems depend on the concept of the attachment complex of a 4-xel $q$ in $I$, which is denoted by $\operatorname{Attach}(q, I)$ and is defined by

$$
\operatorname{Attach}(q, I)=\{f \mid f<q \text { and } f<y \text { for some } y \in I-\{q\}\}
$$

The closed polyhedral set $\bigcup \operatorname{Attach}(q, I)$ is called the attachment set of $q$ in $I$. This set is also given by $\bigcup \operatorname{Attach}(q, I)=q \cap \bigcup(I \backslash\{q\})$. The boundary complex of a 4-xel $q$, denoted by Boundary $(q)$, is the set of all the proper faces of $q$. Evidently, $\operatorname{Attach}(q, I) \subseteq \operatorname{Boundary}(q)$.

For any xel-complex $K$, the Euler characteristic of $K$ is the integer $\chi(K)$ defined by $\chi(K)=c_{0}(K)-c_{1}(K)+c_{2}(K)-c_{3}(K)+c_{4}(K)$, where $c_{n}(K)$ is the number of $n$-xels in $K$. If $\mathcal{P}$ is the union of the xels of a xel-complex $K$, then we define $\chi(\mathcal{P})$ to be $\chi(K)$. It can be shown that if $x$ is any xel then $\chi(x)=1$.

We are now ready to state the above-mentioned essentially discrete sets of necessary and sufficient conditions for a 4 -xel $q$ to be simple.

Theorem 1. Let $q$ be a 4-xel in a $4 D$ binary image $I$. Then $q$ is simple in $I$ if and only if the following all hold:

1. $\bigcup \operatorname{Attach}(q, I)$ is connected and nonempty.

2. UBoundary $(q)-\bigcup \operatorname{Attach}(q, I)$ is connected and nonempty.

3. $\bigcup \operatorname{Attach}(q, I)$ is simply connected.

Theorem 2. Let $q$ be a 4-xel in a $4 D$ binary image $I$. Then $q$ is simple in $I$ if and only if the following all hold:

1. $\cup \operatorname{Attach}(q, I)$ is connected.

2. $\bigcup \operatorname{Boundary}(q)-\bigcup \operatorname{Attach}(q, I)$ is connected.

3. $\chi(\operatorname{Attach}(q, I))=1$.

Theorems 1 and 2 are Theorems 7 and 9 in [6], except that the definition of a simple 4-xel used in that paper might seem to be more stringent than the definition given above: In 6], a 4 -xel $q$ is said to be simple in a 4 D binary image $I$ if $\bigcup \operatorname{Attach}(q, I)$ is a strong deformation retract of $q$.

As is explained in [6], it follows from the main result of [7] that the three conditions of Theorem 1 are equivalent to the three conditions of Theorem 2 . So the two theorems are equivalent.

An elementary proof of the "if" parts of these theorems is given in [6]. A shorter proof can be given using results of algebraic topology - notably Fact 3.4 in 8 (which follows from Corollaries 3.2.5, 1.3.11, and 1.4.10, and Theorem 1.4.11, in [9]) and Corollary 8.3.11 in 10.

Although the "only if" parts of Theorems 1 and 2 are easy to prove if simple 4-xels are defined as in [6], we have to work a little harder to give a proof for the definition of simpleness used in this paper. However, standard techniques of algebraic topology suffice. Indeed, assuming $q$ is simple in $I$ one can use the exact homology sequences of the pairs $(q, \bigcup \operatorname{Attach}(q, I))$ and $(\bigcup I, \bigcup(I \backslash\{q\}))$ together with the excision theorem to deduce that the reduced homology groups 
of $\bigcup \operatorname{Attach}(q, I)$ are all trivial. The three conditions of Theorem 2 follow from this and the Alexander duality theorem.

The definition of simpleness used in [6] is in fact equivalent to the definition used in this paper, because both definitions are equivalent to the three conditions of Theorem [1] or 2] An advantage of the definition we are now using is that it involves only 4-xels (and does not involve their attachment sets).

It may be worth mentioning here that, while Theorem 2 provides an easy way of determining whether or not a 4 -xel in a $4 \mathrm{D}$ binary image is simple, there seems to be no analog of Theorem 2 for 5 - and higher-dimensional images. Indeed, for $n \geq 5$ the authors do not currently have an easy way to determine if the attachment set of an $n$-xel in an $n$-dimensional binary image is simply connected.

\section{MNS Sets and the Main Theorem}

A set $D$ of 4-xels in a 4D binary image $I$ is said to be simple in $I$ if the elements of $D$ can be arranged in a sequence in which each element is simple after all of its predecessors in the sequence have been removed from the image. In particular, the empty set is simple in $I$, and a singleton set $\{q\}$ is simple in $I$ if and only if $q$ is a simple 4-xel of $I$.

Since the deletion of a single simple 4-xel "preserves topology", so does the parallel deletion of a simple set of 4 -xels. More precisely, if $D$ is a simple set of 4 -xels of a $4 \mathrm{D}$ binary image $I$, then it follows from the definition of a simple 4 -xel (and the transitivity of the relation "is a strong deformation retract of") that $\bigcup(I \backslash D)$ is a strong deformation retract of $\bigcup I$.

We are interested in ways of proving that the set of 4 -xels deleted at each iteration of a given parallel thinning algorithm for 4D binary images is always a simple set. (This would imply that the algorithm "preserves topology".) Since a non-simple set must evidently contain a minimal non-simple (MNS) set, one method of proof would be to show that at each iteration no set of 4-xels that all satisfy the algorithm's deletion condition can be an MNS set of the image. (A set $D$ of 4 -xels of $I$ is an MNS set of $I$ if and only if $D$ is a non-simple set of $I$ but every proper subset of $D$ is a simple set of $I$.) In fact, this would show that the set of 4-xels deleted at each iteration is not only simple but also hereditarily simple -i.e., all of its subsets are simple sets of the image. One example of an MNS set in a $4 \mathrm{D}$ binary image is shown in Figure 2,

The next four theorems state fundamental properties of MNS sets. These results were established for 3D binary images in [5]- see Propositions 4.3, 4.5, 4.6 , and 4.7 in that paper - and can be proved for $4 \mathrm{D}$ images in the same way.

Theorem 3. Let $D$ be a nonempty set of 4-xels in a $4 D$ binary image $I$. Then $D$ is MNS in I if and only if the following conditions both hold:

1. Each element $q \in D$ is non-simple in $I \backslash(D \backslash\{q\})$.

2. Each element $q \in D$ is simple in $I \backslash D^{\prime}$ whenever $D^{\prime} \subsetneq D \backslash\{q\}$. 
We say that a set $D$ of 4 -xels can be $M N S$ if there is some 4D binary image $I$ such that $D$ is an MNS set of $I$. We say that a set $D$ of 4 -xels can be $M N S$ without being a component if there is some $4 \mathrm{D}$ binary image $I$ such that $D$ is an MNS set of $I$ and $D$ is not an 80-component of $I$.

Theorem 4. A set of 4 -xels can be MNS only if it is a subset of some $2 \times 2 \times 2 \times 2$ block of sixteen 4 -xels.

Theorem 5. Let $D$ be an MNS set of a $4 D$ binary image $I$, and suppose $D$ is not an 80-component of I. Then every element of $D$ is 80-adjacent to a 4-xel of $I$ that is not in $D$.

Theorem 6. If a set D of 4-xels can be MNS without being a component, then every subset $D^{\prime}$ of $D$ can be MNS without being a component.

We now state our Main Theorem, which identifies all sets of 4-xels that can be MNS, and all such sets that can be MNS without being a component:

Theorem 7 (Main Theorem). Let D be a set of 4-xels. Then:

1. $D$ can be MNS if and only if $D$ is contained in some $2 \times 2 \times 2 \times 2$ block of sixteen 4-xels.

2. $D$ can be MNS without being a component if and only if $D$ is a subset of some $2 \times 2 \times 2$ block of eight 4 -xels.

Note that there are four types of $2 \times 2 \times 2$ block: Such a block could be a $1 \times 2 \times 2 \times 2$, a $2 \times 1 \times 2 \times 2$, a $2 \times 2 \times 1 \times 2$, or a $2 \times 2 \times 2 \times 1$ block.

\section{Proof of the Main Theorem}

\subsection{Useful Results}

The purpose of this subsection is to present three results that will be used in our proof of the Main Theorem.

The first result is the Inclusion-Exclusion Principle for Euler characteristics, which is the following identity:

$$
\chi\left(\bigcup_{i=1}^{n} K_{i}\right)=\sum_{T \subseteq\{1,2, \ldots, n\}, T \neq \emptyset}(-1)^{|T|-1} \chi\left(\bigcap_{i \in T} K_{i}\right)
$$

This holds for arbitrary xel-complexes $K_{1}, K_{2}, \ldots, K_{n}$. The identity follows from the Inclusion-Exclusion Principle for finite sets and the definition of $\chi(K)$.

The second result is the next proposition, which is related to the following lemma:

Lemma 1. Let $\mathcal{P}$ be a union of xels and let $x$ be an edge or a 2-xel such that $x \nsubseteq \mathcal{P}$ and $\chi(x \cap \mathcal{P})=1$. Then one of the following is true: 
1. $x \cap \mathcal{P}$ consists of a single vertex of $x$.

2. $x$ is a 2-xel and $x \cap \mathcal{P}$ is one of the four edges of $x$.

3. $x$ is a 2-xel and $x \cap \mathcal{P}$ is a union of two edges of $x$ that share a vertex.

4. $x$ is a 2-xel and $x \cap \mathcal{P}$ is a union of three of the four edges of $x$.

This lemma is easily verified by considering all possible forms of $x \cap \mathcal{P}$. From the lemma it is not hard to deduce Proposition 1 below, which will be used in section 5.3. We omit the proof of the lemma, but expect most readers will find it intuitively clear that all parts of the proposition are valid in each of the four cases of the lemma.

Proposition 1. Let $q$ be a 4-xel. Let $\mathcal{P}$ be a union of xels in $\operatorname{Boundary}(q)$ and let $x$ be an edge or a 2-xel in $\operatorname{Boundary}(q)$ such that $\chi(x \cap \mathcal{P})=1$. Then:

1. $\mathcal{P}$ is connected if and only if $\mathcal{P} \cup\{x\}$ is connected.

2. $\bigcup$ Boundary $(q) \backslash \mathcal{P}$ is connected if and only if $\bigcup$ Boundary $(q) \backslash(\mathcal{P} \cup\{x\})$ is connected.

3. $\chi(\mathcal{P})=\chi(\mathcal{P} \cup\{x\})$.

The following proposition is the third result. This will save us a lot of casechecking in section 5.2

Proposition 2. Let $q$ be a 4-xel, and let $X$ be any nonempty set of xels in $\operatorname{Boundary}(q)$ that satisfies one of the following two conditions:

A. There is some vertex that belongs to all of the xels in $X$.

B. $X=Y \cup Z$, where $Y \cap Z \neq \emptyset$, there is some vertex that belongs to all of the xels in $Y$, there is some vertex that belongs to all of the xels in $Z$, and no xel in $Y \backslash Z$ intersects a xel in $Z \backslash Y$.

Then $X$ satisfies the following conditions:

1. $\bigcup X$ is connected.

2. $\bigcup$ Boundary $(q) \backslash \bigcup X$ is connected.

3. $\chi(\bigcup X)=1$.

In fact condition $\mathrm{A}$ in this proposition is a special case of condition B (since we may take $Y=Z=X$ in $\mathrm{B}$ ). The proposition follows from Theorem 4.1 in [8]: Condition B implies that $X$ is SN in the sense of [8], and so the theorem implies $\bigcup X$ is contractible. By standard results of algebraic topology (including the Alexander duality theorem) [10], this implies $\bigcup X$ satisfies conditions $1-3$.

\subsection{The "If" Parts of the Main Theorem}

For any 4-xel $q$ in a $4 \mathrm{D}$ binary image $I$, let $A(q, I)=\{q \cap x \mid x \in I \backslash\{q\}\} \backslash\{\emptyset\}$. Note that $\bigcup A(q, I)=\bigcup \operatorname{Attach}(q, I)$.

To show that the "if" part of assertion 1 of the Main Theorem is valid, let $D$ be a subset of a $2 \times 2 \times 2 \times 2$ block of 4 -xels such that $D$ is an 80 -component of a $4 \mathrm{D}$ binary image $I$. We claim $D$ is MNS in $I$. Evidently, $D$ satisfies condition 1 


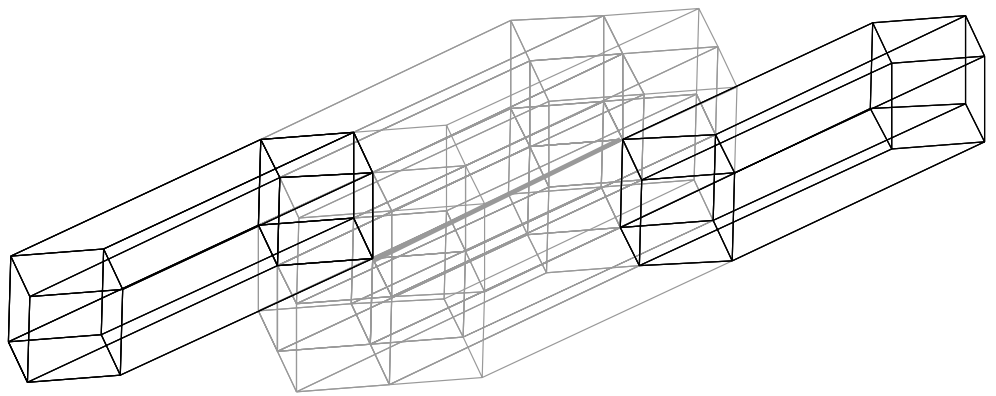

Fig. 2. Let $I$ be the $4 \mathrm{D}$ binary image consisting of ten 4 -xels shown in this figure. Then the $2 \times 2 \times 2$ block of eight 4 -xels in the center constitute an MNS set of $I$. (In the figure a larger scale is used in the direction of the 4 th coordinate axis than in the directions of the other three, so the 4-xels appear to have been stretched in that direction.) Notice that there is an edge, indicated by the thick gray line, which connects the two "end" 4-xels; this edge is shared by the eight central 4 -xels. Let $q$ be any one of those eight 4 -xels. Then it is easy to deduce from Theorem 2 and Proposition 2 that $q$ is simple in the image that remains after any proper subset of the other seven central 4 -xels is deleted from $I$. But if we delete all seven of the other central 4-xels from $I$ then $q$ is non-simple in the remaining image (which consists just of $q$ and the two end 4-xels). Hence, by Theorem 3, the central block of eight 4-xels is MNS in $I$ as we claimed.

of Theorem 3. It remains to show that $D$ also satisfies condition 2 of Theorem 3 Let $q \in D$ and let $I^{\prime}$ be obtained from $I$ by deleting any proper subset of the other elements of $D$. We need to show that $q$ is simple in $I^{\prime}$. Let $X=A\left(q, I^{\prime}\right)$. As $D$ is contained in a $2 \times 2 \times 2 \times 2$ block of 4 -xels, the central vertex of that block belongs to all the 4 -xels in $D$ and hence to all the xels in $X$. So, since $\bigcup X=\bigcup \operatorname{Attach}\left(q, I^{\prime}\right)$, it follows from Proposition 2 that the three conditions of Theorem 2 hold with $I^{\prime}$ in place of $I$. Thus $q$ is simple in $I^{\prime}$, as required.

To show that the "if" part of assertion 2 is also valid, let $I$ be a $2 \times 2 \times 2 \times 3$ block of 4 -xels and let $D$ be its central $2 \times 2 \times 2 \times 1$ block (which is clearly not an 80-component of $I$ ). We claim $D$ is MNS in $I$. If we can prove this then, by Theorem 6, the "if" part of assertion 2 is valid. By symmetry we may assume that $I=\left\{i_{1} \times i_{2} \times i_{3} \times i_{4} \mid i_{1}, i_{2}, i_{3} \in\{[0,1],[1,2]\}, i_{4} \in\{[0,1],[1,2],[2,3]\}\right\}$ so that $D=\left\{i_{1} \times i_{2} \times i_{3} \times[1,2] \mid i_{1}, i_{2}, i_{3} \in\{[0,1],[1,2]\}\right\}$. Then $D$ clearly satisfies condition 1 of Theorem 3 To show that $D$ also satisfies condition 2 of Theorem 3 let $q \in D$ and let $I^{\prime}$ be obtained from $I$ by deleting any proper subset of the other seven 4-xels in $D$. We need to show that $q$ is simple in $I^{\prime}$.

Let $D^{-}=\left\{i_{1} \times i_{2} \times i_{3} \times[0,1] \mid i_{1}, i_{2}, i_{3} \in\{[0,1],[1,2]\}\right\}$ and let $D^{+}=$ $\left\{i_{1} \times i_{2} \times i_{3} \times[2,3] \mid i_{1}, i_{2}, i_{3} \in\{[0,1],[1,2]\}\right\}$.

Let $X=A\left(q, I^{\prime}\right)$. Since $I^{\prime} \subseteq D^{-} \cup D \cup D^{+}$, we have $X=Y \cup Z$, where $Y=A\left(q, I^{\prime} \cap\left(D \cup D^{-}\right)\right)$and $Z=A\left(q, I^{\prime} \cap\left(D \cup D^{+}\right)\right)$. Since $D \cup D^{-}=$ $\left\{i_{1} \times i_{2} \times i_{3} \times i_{4} \mid i_{1}, i_{2}, i_{3}, i_{4} \in\{[0,1],[1,2]\}\right\}$, the vertex $(1,1,1,1)$ belongs to all the 4-xels in $D \cup D^{-}$and hence to all the xels in $Y$. Similarly, the vertex $(1,1,1,2)$ 
belongs to all the xels in $Z$. Moreover, $Y \cap Z=A\left(q, I^{\prime} \cap D\right) \neq \emptyset$ because at least one element of $D \backslash\{q\}$ is in $I^{\prime}$. Also, no xel in $Y \backslash Z=A\left(q, I^{\prime} \cap D^{-}\right)$intersects a xel in $Z \backslash Y=A\left(q, I^{\prime} \cap D^{+}\right)$. Since $\bigcup X=\bigcup \operatorname{Attach}\left(q, I^{\prime}\right)$, it follows from Proposition 2 that the three conditions of Theorem 2 hold with $I^{\prime}$ in place of $I$, which implies $q$ is simple in $I^{\prime}$, as required.

\subsection{The "Only If" Parts of the Main Theorem}

The "only if" part of assertion 1 is just Theorem 4. To prove the "only if" part of assertion 2, let $S$ be any MNS set of a 4D binary image $I$. By Theorem 4 , $S$ is contained in some $2 \times 2 \times 2 \times 2$ block.

A set $T$ of 4 -xels that is contained in some $2 \times 2 \times 2 \times 2$ block will be called a spanning set if there is no $2 \times 2 \times 2$ block that contains $T$.

We now suppose that our MNS set $S$ is a minimal spanning set-i.e., we suppose $S$ is a spanning set but no proper subset of $S$ is a spanning set-and deduce that $S$ must be an 80 -component of $I$. This will show that no minimal spanning set can be MNS without being a component, which (by Theorem 6) is enough to establish the "only if" part of assertion 2 of the Main Theorem, since every spanning set contains a minimal spanning set.

For any two 4-xels $p$ and $q$ let $p-q$ denote the vector from the centroid of $q$ to the centroid of $p$. We define the $l_{1}$-diameter of $S$ to be $\max _{p, q \in S}\|p-q\|_{1}$, where $\|\mathbf{v}\|_{1}$ is the $l_{1}$-norm of the vector $\mathbf{v}$ (i.e., the sum of the absolute values of the four components of $\mathbf{v}$ ). Since $S$ is a spanning set, the $l_{1}$-diameter of $S$ is at least 2 , and is therefore equal to 2,3 , or 4 .

\section{Case 1: The $l_{1}$-Diameter of $S$ Is 4}

In this case $S=\{q, a\}$ for some 4 -xels $q$ and $a$ such that $\|q-a\|_{1}=4$. Note that $q \cap a$ consists of just one vertex, $v$ say. Let $\mathcal{P}=\bigcup \operatorname{Attach}(q, I \backslash\{a\})$, so that $\cup \operatorname{Attach}(q, I)=\mathcal{P} \cup\{v\}$. Since $S$ is MNS in $I$, it follows from Theorem 3 that $q$ is non-simple in $I \backslash\{a\}$ but $q$ is simple in $I$. The latter and Theorem 2 imply $\mathcal{P} \cup\{v\}=\bigcup \operatorname{Attach}(q, I)$ is connected, and so either $v \in \mathcal{P}$ or $\mathcal{P}=\emptyset$. But $v \in \mathcal{P}$ would imply $\bigcup \operatorname{Attach}(q, I)=\mathcal{P} \cup\{v\}=\mathcal{P}=\bigcup \operatorname{Attach}(q, I \backslash\{a\})$, which (by Theorem [2) would make it impossible for $q$ to be simple in $I$ but non-simple in $I \backslash\{a\}$. Hence $\mathcal{P}=\emptyset$ and so, by Theorem $5, S$ is an 80-component of $I$.

\section{Case 2: The $l_{1}$-Diameter of $S$ Is 3}

In this case it is readily confirmed that $S=\{q, a, b\}$ for some 4 -xels $q, a$, and $b$ such that $\|q-a\|_{1}=\|q-b\|_{1}=3$ and $\|a-b\|_{1}=2$. Let $q \cap a=e_{a}$ and $q \cap b=e_{b}$. Then $e_{a}$ and $e_{b}$ are edges and $e_{a} \cap e_{b}$ consists of just a vertex.

Let $\mathcal{P}=\bigcup \operatorname{Attach}(q, I \backslash\{a, b\}), \operatorname{so} \bigcup \operatorname{Attach}(q, I)=\mathcal{P} \cup e_{a} \cup e_{b}, \bigcup \operatorname{Attach}(q, I \backslash$ $\{a\})=\mathcal{P} \cup e_{b}$, and $\bigcup \operatorname{Attach}(q, I \backslash\{b\})=\mathcal{P} \cup e_{a}$. Since $S$ is MNS in $I$, it follows from Theorem 3 that $q$ is non-simple in $I \backslash\{a, b\}$ but $q$ is simple in $I$, in $I \backslash\{a\}$, and in $I \backslash\{b\}$. So it follows from Theorem [2 and Proposition 1 that neither $\chi\left(\mathcal{P} \cap e_{a}\right)$ nor $\chi\left(\mathcal{P} \cap e_{b}\right)$ is equal to 1 . 
$\operatorname{As} \bigcup \operatorname{Attach}(q, I)=\mathcal{P} \cup e_{a} \cup e_{b}, \bigcup \operatorname{Attach}(q, I \backslash\{a\})=\mathcal{P} \cup e_{b}, \bigcup \operatorname{Attach}(q, I \backslash$ $\{b\})=\mathcal{P} \cup e_{a}$, and $\chi\left(e_{a}\right)=\chi\left(e_{b}\right)=\chi\left(e_{a} \cap e_{b}\right)=1$, it follows from Theorem 2 and the Inclusion-Exclusion Principle for Euler characteristics that

$1=\chi\left(\mathcal{P} \cup e_{a}\right)=\chi(\mathcal{P})+1-\chi\left(\mathcal{P} \cap e_{a}\right)$

$1=\chi\left(\mathcal{P} \cup e_{b}\right)=\chi(\mathcal{P})+1-\chi\left(\mathcal{P} \cap e_{b}\right)$

$1=\chi\left(\mathcal{P} \cup e_{a} \cup e_{b}\right)=\chi(\mathcal{P})+1+1-\chi\left(\mathcal{P} \cap e_{a}\right)-\chi\left(\mathcal{P} \cap e_{b}\right)-1+\chi\left(\mathcal{P} \cap e_{a} \cap e_{b}\right)$

and therefore $\chi(\mathcal{P})=\chi\left(\mathcal{P} \cap e_{a}\right)=\chi\left(\mathcal{P} \cap e_{b}\right)=\chi\left(\mathcal{P} \cap e_{a} \cap e_{b}\right)$.

So, since neither $\chi\left(\mathcal{P} \cap e_{a}\right)$ nor $\chi\left(\mathcal{P} \cap e_{b}\right)$ is equal to $1, \chi\left(\mathcal{P} \cap e_{a} \cap e_{b}\right) \neq 1$. Thus $\mathcal{P} \cap e_{a} \cap e_{b}=\emptyset$ (since $e_{a} \cap e_{b}$ consists of just a vertex) and $\chi\left(\mathcal{P} \cap e_{a} \cap e_{b}\right)=0$. Therefore $\chi(\mathcal{P})=\chi\left(\mathcal{P} \cap e_{a}\right)=\chi\left(\mathcal{P} \cap e_{b}\right)=0$, so $\mathcal{P} \cap e_{a}=\emptyset$. Now if $\mathcal{P} \neq \emptyset$ then $\mathcal{P} \cup e_{a}$ is disconnected, which contradicts Theorem 2 because $\mathcal{P} \cup e_{a}=$ $\bigcup \operatorname{Attach}(q, I \backslash\{b\})$ and $q$ is simple in $I \backslash\{b\}$. Hence $\mathcal{P}=\emptyset$ and so, by Theorem 5 $S$ is an 80-component of $I$.

\section{Case 3: The $l_{1}$-Diameter of $S$ Is 2}

In this case it is quite easy to verify that $S=\{q, a, b, c\}$, where $\|x-y\|_{1}=2$ for all distinct $x$ and $y$ in $S$. Let $q \cap a=f_{a}, q \cap b=f_{b}$, and $q \cap c=f_{c}$. Then $f_{a}, f_{b}$, and $f_{c}$ are 2-xels, every pair of them share an edge, and $f_{a} \cap f_{b} \cap f_{c}$ consists of just a vertex.

Let $\mathcal{P}=\bigcup \operatorname{Attach}(q, I \backslash\{a, b, c\})$, so that $\bigcup \operatorname{Attach}(q, I \backslash\{a, b\})=\mathcal{P} \cup f_{c}$. Since $S$ is MNS in $I$, it follows from Theorem 3 that $q$ is non-simple in $I \backslash\{a, b, c\}$ but $q$ is simple in $I, I \backslash\{c\}, I \backslash\{b, c\}$, and $I \backslash\{a, b\}$. So it follows from Theorem 2 and Proposition 1 that $\chi\left(\mathcal{P} \cap f_{c}\right) \neq 1$

Since $\cup \operatorname{Attach}(q, I \backslash\{b, c\})=\mathcal{P} \cup f_{a}$, it follows from Theorem 2 the fact that $\chi(x)=1$ for any xel $x$, and the Inclusion-Exclusion Principle for Euler characteristics that $1=\chi\left(\mathcal{P} \cup f_{a}\right)=\chi(\mathcal{P})+1-\chi\left(\mathcal{P} \cap f_{a}\right)$. Hence $\chi(\mathcal{P})=\chi\left(\mathcal{P} \cap f_{a}\right)$. By symmetrical arguments we must have

$$
\chi(\mathcal{P})=\chi\left(\mathcal{P} \cap f_{a}\right)=\chi\left(\mathcal{P} \cap f_{b}\right)=\chi\left(\mathcal{P} \cap f_{c}\right)
$$

Similarly, since $\bigcup \operatorname{Attach}(q, I \backslash\{c\})=\mathcal{P} \cup f_{a} \cup f_{b}$, we have $1=\chi\left(\mathcal{P} \cup f_{a} \cup f_{b}\right)=$ $\chi(\mathcal{P})+1+1-\chi\left(\mathcal{P} \cap f_{a}\right)-\chi\left(\mathcal{P} \cap f_{b}\right)-1+\chi\left(\mathcal{P} \cap f_{a} \cap f_{b}\right)$ and so by eqn. (2) we have $\chi(\mathcal{P})=\chi\left(\mathcal{P} \cap f_{a} \cap f_{b}\right)$. By symmetrical arguments we must have

$$
\chi(\mathcal{P})=\chi\left(\mathcal{P} \cap f_{a} \cap f_{b}\right)=\chi\left(\mathcal{P} \cap f_{b} \cap f_{c}\right)=\chi\left(\mathcal{P} \cap f_{a} \cap f_{c}\right)
$$

Again, since $\cup \operatorname{Attach}(q, I)=\mathcal{P} \cup f_{a} \cup f_{b} \cup f_{c}$, we have $1=\chi\left(\mathcal{P} \cup f_{a} \cup f_{b} \cup f_{c}\right)=$ $\chi(\mathcal{P})+1+1+1-\chi\left(\mathcal{P} \cap f_{a}\right)-\chi\left(\mathcal{P} \cap f_{b}\right)-\chi\left(\mathcal{P} \cap f_{c}\right)-1-1-1+\chi\left(\mathcal{P} \cap f_{a} \cap\right.$ $\left.f_{b}\right)+\chi\left(\mathcal{P} \cap f_{b} \cap f_{c}\right)+\chi\left(\mathcal{P} \cap f_{a} \cap f_{c}\right)+1-\chi\left(\mathcal{P} \cap f_{a} \cap f_{b} \cap f_{c}\right)$ and so by equations (2) and (3) we have:

$$
\chi(\mathcal{P})=\chi\left(\mathcal{P} \cap f_{a} \cap f_{b} \cap f_{c}\right)
$$

Recalling that $\chi\left(\mathcal{P} \cap f_{c}\right) \neq 1$, we see that equations (2) and (4) imply $\chi(\mathcal{P} \cap$ $\left.f_{a} \cap f_{b} \cap f_{c}\right) \neq 1$. Thus $\mathcal{P} \cap f_{a} \cap f_{b} \cap f_{c}=\emptyset$ (since $f_{a} \cap f_{b} \cap f_{c}$ consists of just 
a vertex) and $\chi\left(\mathcal{P} \cap f_{a} \cap f_{b} \cap f_{c}\right)=0$. Therefore, by equations (2), (3), and (4), the 2-xel $f_{a}$ satisfies $\chi\left(\mathcal{P} \cap f_{a}\right)=\chi\left(\mathcal{P} \cap f_{a} \cap f_{b}\right)=0$. Since $\chi\left(\mathcal{P} \cap f_{a}\right)=0$, $\mathcal{P} \cap f_{a}$ either is empty or is the union of the four edges of $f_{a}$. But the latter is impossible because $f_{a} \cap f_{b}$ is an edge of $f_{a}$ that is not contained in $\mathcal{P}$ (since $\left.\chi\left(\mathcal{P} \cap f_{a} \cap f_{b}\right)=0\right)$. Hence $\mathcal{P} \cap f_{a}=\emptyset$. Now if $\mathcal{P} \neq \emptyset$ then $\mathcal{P} \cup f_{a}$ is disconnected, which contradicts Theorem 2 because $\mathcal{P} \cup f_{a}=\bigcup \operatorname{Attach}(q, I \backslash\{b, c\})$ and $q$ is simple in $I \backslash\{b, c\}$. So $\mathcal{P}=\emptyset$ and, by Theorem 5 , $S$ is an 80-component of $I$.

\section{Concluding Remarks}

We have identified all types of sets of 4-xels that can be minimal non-simple (MNS) in a 4D binary image, and all types that can be MNS without being an 80-component of the image, when 4D 80-connectedness is used on 4-xels in the image and 4D 8-connectedness on 4-xels in its complement. This work is based on a characterization of simple 4-xels that was given in [6], and the InclusionExclusion Principle for Euler characteristics.

\section{References}

1. Ronse, C.: Minimal test patterns for connectivity preservation in parallel thinning algorithms for binary digital images. Discrete Applied Mathematics 21 (1988) $67-79$

2. Hall, R.W.: Tests for connectivity preservation for parallel reduction operators. Topology and Its Applications 46 (1992) 199-217

3. Ma, C.M.: On topology preservation in 3D thinning. CVGIP: Image Understanding 59 (1994) 328-339

4. Gau, C.J., Kong, T.Y.: Minimal nonsimple sets of voxels in binary images on a facecentered cubic grid. International Journal of Pattern Recognition and Artificial Intelligence 13 (1999) 485-502

5. Kong, T.Y.: On topology preservation in $2 \mathrm{D}$ and $3 \mathrm{D}$ thinning. International Journal of Pattern Recognition and Artificial Intelligence 9 (1995) 813-844

6. Kong, T.Y.: Topology preserving deletion of 1's from 2-, 3- and 4-dimensional binary images. In Ahronovitz, E., Fiorio, C., eds.: Discrete Geometry for Computer Imagery: 7th International Workshop (DGCI '97, Montpellier, France, December 1997), Proceedings. Springer (1997) 3-18

7. Kong, T.Y., Roscoe, A.W.: Characterizations of simply-connected finite polyhedra in 3-space. Bulletin of the London Mathematical Society 17 (1985) 575-578

8. Saha, P.K., Kong, T.Y., Rosenfeld, A.: Strongly normal sets of tiles in $N$ dimensions. Electronic Notes in Theoretical Computer Science 46 (2001)

URL: http://www.elsevier.nl/locate/entcs/volume46.html

9. Spanier, E.H.: Algebraic Topology. Springer (1989)

10. Maunder, C.R.F.: Algebraic Topology. Dover (1996) 